\title{
Variants in the APOA5 gene region and the response to combination therapy with statins and fenofibric acid in a randomized clinical trial of individuals with mixed dyslipidemia
}

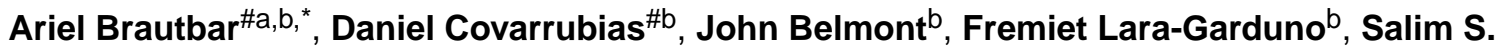 \\ Virani $^{a, c, d}$, Peter H. Jones ${ }^{a, c}$, Suzanne M. Leal ${ }^{b}$, and Christie M. Ballantyne ${ }^{a, b, c}$ \\ aSection of Cardiovascular Research, Department of Medicine, Baylor College of Medicine, \\ Houston, TX, USA

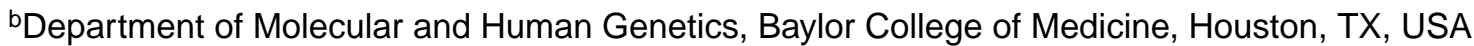 \\ cMethodist DeBakey Heart and Vascular Center and Baylor College of Medicine, Houston, TX, \\ USA \\ dStaff Cardiologist, Michael E. DeBakey Veterans Affairs Medical CenterAssistant Professor, \\ Section of Atherosclerosis and Vascular Medicine, Department of Medicine, Houston, TX, USA \\ \# These authors contributed equally to this work.
}

\section{Abstract}

Objective: Atherogenic dyslipidemia is highly associated with coronary heart disease and is characterized by elevated triglycerides (TG), low high-density lipoprotein cholesterol (HDL-C), and elevated low-density lipoprotein cholesterol (LDL-C). The combination of statins and fibrates is a common modality to treat individuals with atherogenic dyslipidemia.

We sought to identify single nucleotide polymorphisms (SNPs) associated with HDL-C, TG, and apolipoprotein A1 (ApoA-I) response to combination therapy with statins and fenofibric acid (FA) in individuals with atherogenic dyslipidemia.

Methods: 2228 individuals with mixed dyslipidemia who were participating in a multicenter, randomized, double-blind, active-controlled study comparing FA alone, in combination with a statin, or statin alone for a 12-week period, were genotyped for 304 candidate SNPs. A multivariate linear regression analysis for percent change in HDL-C, ApoA-I and TG levels was performed.

Results: SNPs in the apolipoprotein (APO) A5-ZNF259 region rs3741298 $\left(P=1.8 \times 10^{-7}\right)$, rs964184 $\left(P=3.6 \times 10^{-6}\right)$, rs651821 $\left(P=4.5 \times 10^{-5}\right)$, and rs10750097 $\left(P=1 \times 10^{-4}\right)$, were significantly associated with HDL-C response to combination therapy with statins and FA, with a

"Corresponding author at: Baylor College of Medicine, 6565 Fannin Street, Suite B160, MS-A601, Houston, TX 77030, USA. Tel.: +1 713798 5321; fax: +1 713798 7885. brautbar@bcm.edu.

Disclosures

Peter H. Jones and Christie M. Ballantyne are consultants and have received honorariums from Abbott; the rest of the authors have no disclosures.

Appendix A. Supplementary data

Supplementary data associated with this article can be found, in the online version, at doi:10.1016/j.atherosclerosis.2011.08.015. 
similar association identified for ApoA-I. A haplotype composed of the minor alleles of SNPs rs3741298, rs964184, and rs10750097, was associated with a positive response to statins and FA $\left(P=8.7 \times 10^{-7}\right)$ and had a frequency of $18 \%$ in the study population.

Conclusion: In a population with atherogenic dyslipidemia, common SNPs and haplotypes within the APOA5-ZNF259 region are highly associated with HDL-C and ApoA-I response to combination therapy with statins and FA.

\section{Keywords}

Genetic variants; Combination therapy; Mixed dyslipidemia

\section{Introduction}

Elevated triglycerides (TG) and low levels of high-density lipoprotein cholesterol (HDL-C), with or without high levels of low-density lipoprotein cholesterol (LDL-C), characterize a dyslipidemia that places people at high risk for coronary heart disease (CHD). This phenotype, referred to as atherogenic dyslipidemia or mixed dyslipidemia, occurs frequently among individuals with insulin resistance such as the metabolic syndrome and type 2 diabetes. Monotherapy with statins is seldom sufficient to improve all lipid levels, including achieving non-HDL-C targets, in those with atherogenic dyslipidemia, and the addition of other agents such as niacin or a fibrate such as fenofibric acid (FA) is often required. Although recent clinical trials have questioned the utility of fenofibrate alone or in combination with statins to lower CHD events, individuals with mixed dyslipidemia in the Action to Control Cardiovascular Risk in Diabetes (ACCORD) trial had incremental benefit in CHD event reduction from combination of fenofibrate and statin therapy compared to statin monotherapy [1-3]. A similar benefit was observed in individuals with mixed dyslipidemia participating in the Fenofibrate Intervention and Event Lowering in Diabetes (FIELD) study with a reduction in CHD events following fenofibrate treatment $[2,3]$.

FA is a PPARA receptor agonist which modulates multiple downstream targets including ABCA1 and apolipoproteins AI, AII, and CIII. The lipid response to fenofibrate treatment varies both by individual and by lipid phenotypes (e.g., normo-lipidemic vs. mixed dyslipidemia), and its specific mechanism of action supports the notion that genetic variation may have an important effect on determining individual response to therapy. In previous studies, a few polymorphisms have been identified that are associated with TG and HDL-C response to fenofibrate therapy. However, most of these studies did not examine individuals with mixed dyslipidemia who may be, the individuals most likely to benefit from the addition of fibrates to statins, and none of the prior studies have examined combination therapy of fibrates and statins [4-6].

In this study, we examined the association of single nucleotide polymorphisms (SNPs) and the response to the combination of FA and statins in subjects with mixed dyslipidemia who enrolled in a randomized, double-blind, active-controlled clinical trial program. We hypothesized that common genetic variants will have a significant effect on the lipid response to combination therapy with FA and statins in individuals with mixed dyslipidemia. 


\section{Methods}

\subsection{Study population}

The study population included men and women who participated in a program which included 3 concurrent prospective, randomized, double-blind, phase- 3 studies designed to examine the efficacy for a new FA formulation (fenofibric acid, TriLipix, Abbott, Abbott Park, IL, USA). A detailed description of the study design has been published elsewhere [7,8]. In brief, inclusion criteria included TG $\geq 150 \mathrm{mg} / \mathrm{dL}$, HDL-C $<40 \mathrm{mg} / \mathrm{dL}$ for men or $<50 \mathrm{mg} / \mathrm{dL}$ for women, and LDL-C $\geq 130 \mathrm{mg} / \mathrm{dL}$. In the three studies, participants were randomized into groups that received either FA alone, statin alone, or combination of FA and statins. Participants had a washout period of 6 weeks in which no lipid-modifying therapy was given. A 12-week treatment phase followed the washout period, and lipid measurements were obtained at the beginning and end of the treatment period. Additional details regarding the clinical study design are included in the Supplement. Multidimensional scaling revealed no population substructure in the combined study population.

\subsection{Selection of genes and SNPs}

Genes were selected based on their involvement in HDL-C and TG metabolism [9,10]. In order to evaluate most of the common variations in each gene, tag SNPs were selected using the Genome Variation Server (http://gvs.gs.washington.edu/GVS/) based on the following criteria: HapMap-CEU dataset, $r^{2} \geq 8$ for each bin, minor allele frequency $\geq 1 \%$, and a 4-10 $\mathrm{kB}$ margin from gene bound ary. Additional SNPs were added based on published genomewide association studies for associations with HDL-C and TG [11-14]. A total of 350 SNPs to be tested for therapy response were selected, and 34 SNPs with inter-ethnic difference in allele frequencies were added to aid in correcting for potential population stratification within the European-American population [15]. The list of SNPs and the reason for their inclusion is detailed in Supplemental Table 1. SNPs were genotyped using Golden Gate chemistry on an Illumina Bead Express system (Igenix, Seattle, WA). Samples with call rate $<90 \%$ were excluded. SNPs were excluded if they had a call rate $<95 \%$, showed evidence of deviation from Hardy-Weinberg equilibrium (HWE) at $P<0.001$ using the exact test, or were monomorphic.

\subsection{Statistical analysis}

Statistical analysis was performed using PLINK (version v1.07, http:// pngu.mgh.harvard.edu/purcell/plink/) [16]. In order to maximize statistical power, treatment groups of the original study design were collapsed into 3 major therapy groups: FA alone ( $n$ $=341)$; statin alone $(n=864)$; and, combination therapy of statins and FA ("CG" group, $n=$ 674). Analysis was restricted to the European-American population in order to avoid increased type I and type II errors due to population stratification. Samples designated "European-American" were selected and subjected to multidimensional scaling analysis. Uncorrelated SNPs were identified by pruning out those with pair-wise linkage disequilibrium $r^{2}>0.5$. The remaining SNPs (including all 34 ancestry-informative SNPs) were then used to cluster the individuals based on identity by state (IBS) analysis. The resulting IBS data were used in multidimensional scaling, and 4 components were saved. All 
procedures were carried out in PLINK. No significant structure in the sample was detected (Supplemental Fig. 1).

The initial discovery phase analysis was performed using linear regression including age and sex as covariates in order to identify the SNPs with the strongest association with percent change of HDL-C, TG, or ApoA-I in each of the groups after treatment. The second phase of the analysis included linear regression for three most significant SNPs and with the highest population frequency identified in the "CG" group. The outcome was percent change, and the covariates were age, sex, body mass index (BMI), smoking, and diabetes. Percent change was defined by the difference in trait level before and after treatment, standardized by the before-treatment level. Association testing for each examined trait with the candidate SNPs was considered a separate hypothesis. A probability value $<1.7 \times 10^{-4}$ was considered significant after Bonferroni correction for the number of SNPs tested (304 after exclusions). Permutation analysis as implemented in PLINK was performed to verify the significance of the associations. Haplotypes were inferred using the expectation-maximization algorithm method. Only haplotypes with inferred frequency $>0.05$ were analyzed. Risk haplotypes were identified based on discovered SNPs in the "CG" treatment group. Multivariate regression analysis of haplotype and covariates was performed, and the partial regression coefficients were estimated for each parameter. The Wald test $P$-value was reported for each haplotype. Covariates age, sex, BMI, smoking, and diabetes were included in the haplotype analysis.

\section{Results}

Of the 384 genotyped SNPs, the following were excluded from the analysis: monomorphic SNPs $(n=2)$, SNPs with minor allele frequency (MAF) $<5 \%$ and missing $>1 \%$ or MAF $>5 \%$ and missing $>5 \%(n=14)$, and those with HWE $<0.001(\mathrm{n}=30)$. After excluding the 34 SNPs selected for stratification analysis, 304 SNPs remained for hypothesis testing.

From a total sample size of 2684 individuals, 456 samples were excluded. Samples were excluded due to a total call rate $<95 \%(n=66)$, duplicated DNA samples $(n=64)$, and ethnicity other than European $(n=326)$. Mean increase in HDL-C in the combination therapy group was $18.3 \%$, and the mean TG decrease was $44.7 \%$ which is similar to other studies examining the combination of statins and fenofibrates in individuals with phenotypes similar to mixed dyslipidemia which showed an increase of 16-23\% in HDL-C and a decrease of $29-50 \%$ in TG [17-20].

There was no significant difference in baseline characteristics between the three treatment groups (Supplemental Table 2).

There were a number of SNPs which had significant associations with HDL-C and ApoA-I percent change following FA treatment. The strongest associations were detected in the ZNF259-APOA5 gene region on chromosome 11 (Table 1 and Supplemental Fig. 2).

The top three SNPs identified in the "CG" group for HDLC percent change were rs3741298 $\left(P=1.8 \times 10^{-7}\right)$, rs964184 $\left(P=3.6 \times 10^{-6}\right)$, and rs651821 $\left(P=4.5 \times 10^{-5}\right)$. The top three SNPs identified in the "CG" group for ApoA-I percent change were rs3741298 $(P=$ 
$\left.2 \times 10^{-4}\right)$, rs964184 $\left(P=3 \times 10^{-4}\right)$, and rs6589567 $\left(P=1 \times 10^{-4}\right)$ as detailed in Supplemental Table 4 .

The significant association of the 3 SNPs with response to therapy for HDL-C or ApoA-I persisted when additional covariates (age, sex, BMI, diabetes, smoking, TG) were included in the regression model (Supplemental Table 3); similarly, the addition of either baseline TG levels, percent change in TG levels, or post therapy (final) TG levels did not have a large effect on the significance of the associations (Supplemental Table 5).

The mean percent change in HDL-C following the combination of statins and FA therapy in the CG group for rs3741298, rs964184 and rs10750097 ranged from 15 to $15.9 \%$ in wild type, 20.4 to $22.9 \%$ in heterozygotes, and 28.3 to $31.7 \%$ in minor allele homozygotes (Table 2 and Supplemental Fig. 3a). The mean percent change in ApoA-I following FA therapy in the CG group for the same SNPs ranged from 7.2 to $8.2 \%$ in the wild type, 11 to $12.4 \%$ in heterozygotes, and 15.2 to $18.5 \%$ in minor allele homozygotes (Table 2 and Supplemental Fig. 3b).

When applying the ANOVA statistical metrics to examine differences in therapy response in the FA-only, CG, and statin-only treatment groups, there were significant differences in APOA-I and HDL-C response between genotypes in the CG group (Table 2). However, there were no significant differences in HDL-C, TG, and APOA-1 therapy response (after correction for multiple testing) between rs3741298, rs964184 and rs10750097 genotypes within the FA-only and statin-only treatment groups (Supplemental Table 6).

When examining other treatment groups for SNPs rs3741298, rs964184, and rs10750097, the statistical significance of the association with percent change in HDL-C or APOAI decreased or was absent in the FA-only group. This may have been related to the small sample size of the FA-only group as it was less than half the size of the "CG" group. None of the 304 SNPs that were tested were associated with percent change of HDL-C or ApoA-I in the statin-only treatment group.

When examining the entire European-American study population, none of the SNPs associated with percent change with HDL-C or ApoA-I had a significant association with baseline levels of either trait before therapy. However, rs3741298 $\left(P=3.3 \times 10^{-5}\right)$ and rs964184 $\left(P=2.4 \times 10^{-7}\right)$ were highly associated with baseline TG levels, while other SNPs that were associated with percent change of either ApoA-I or HDL-C, had somewhat weaker associations with baseline TG levels (Supplemental Table 7).

Empirical estimation of P-values using permutation for the association analysis of rs3741298, rs964184 and rs10750097 with HDL-C and ApoA-I percent change confirmed the statistical significance observed in the discovery phase (Supplemental Table 8). There was no significant difference between baseline HDL-C or ApoA-I mean levels across the three possible genotypes for each SNP: rs3741298, rs964184 and rs10750097. Following combination therapy with FA and statins, the means for both traits became significantly different across genotypes (Supplemental Table 9). On the other hand, there was a significant difference across genotypes for each of the 3 SNPs for baseline TG level which disappeared after therapy (Supplemental Table 9). 
Analysis for SNP associations by way of regression analysis for an additive model with TG percent change adjusted for age and sex did not reveal any significant associations after correcting for multiple testing in the discovery phase (Table 2 and Supplemental Fig. 3c).

Lai et al. showed an association between rs3135506 in the APOA5 gene with TG and HDL$\mathrm{C}$ levels with both baseline levels and change in levels following therapy. There was no association between rs3135506 and post therapy TG and HDL-C levels in that report [4]. We have examined rs3135506 in the current study as well. The collapsed heterozygous and minor allele homozygous geno-type frequency for rs3135506 observed by Lai et al. was $11.2 \%$, while the frequency for the same observed in our study was $17.2 \%$. They showed that rs3135506 was associated with both TG and HDL-C at baseline and not associated after fenofibrate therapy. When we examined the rs3135506 genotype in our study population, there was no association with baseline HDL-C level. However, there was an association with baseline ApoA-I $(P=0.001)$. Following FA treatment, rs3135506 showed a significant association with HDL-C $(P=0.0009)$ and a weaker association with ApoA-I $(P=0.01)$. Similar to the findings by Lai et al., rs3135506 had an association with TG at baseline ( $P=$ $0.0002)$ which no longer held after therapy. The linkage disequilibrium (LD) between rs3135506 and rs3741298 $\left(r^{2}=0.28\right)$, rs964184 $\left(r^{2}=0.4\right)$, and $\operatorname{rs} 10750097\left(r^{2}=0.29\right)$ in our study population, suggested that the mechanism by which these SNPs and the rs3135506 are associated with therapy response may be different.

\subsection{Haplotype analysis}

We examined the haplotypes composed of SNPs rs3741298, rs964184, and rs10750097. In the "CG" group, the CGG haplotype (frequency $=18 \%$ ), which consists on the minor allele of each marker, was associated with a positive HDL-C response $\left(P=8.7 \times 10^{-7}\right)$ to statin FA combination therapy Supplemental Table 10a), and modestly (0.03) associated with a negative TG response to statin FA combination therapy (Supplemental Table 10b).

The $A P O A 5$ gene region has been previously studied; and haplotypes composed of SNPs rs3135506, rs651821, rs662799, rs2266788, and rs2072560, previously analyzed [21,22]. In order to compare our results with previous analyses, we examined haplo-types that involved these markers. We genotyped 3 SNPs included in this haplotype (rs3135506, rs651821, and rs662799). We also genotyped rs2075290 which is in perfect linkage disequilibrium with rs2266788 $\left(r^{2}=1\right)$ in the HapMap database, but did not directly genotype rs226678 or rs2072560. The haplotypes, GCGG, AGAA, and ACAA, were associated with a positive HDL-C response to statin FA combination therapy in the CG treatment group with a combined frequency of $\sim 25 \%\left(P=2.5 \times 10^{-4}, P=0.004\right.$, and $P=8.9 \times 10^{-7}$ respectively $)$ (Supplemental Table 11a) while none of thesehaplo-types had a significant association with TG levels response to statin FA combination therapy in the CG group (Supplemental Table 11b).

\section{Discussion}

In this study, we have identified a number of genetic variants in the APOA5-ZNF259 gene region which are highly associated with percent change in HDL-C and ApoA-I in response to combination therapy with FA and statins in subjects with mixed dyslipidemia baseline. 
The $18.6 \%$ increase in HDL-C following statin and FA combination therapy was larger than that reported in previous studies examining individuals without mixed dyslipidemia in which HDLC increase ranged between 6 and $8 \%$ but similar to other studies examining populations with a mixed dyslipidemia like phenotype in which an increase of 16-23\% in HDL-C was demonstrated [17-20].

Based on previous publications, the addition of fibrates to statins does not simply result in an additive effect of both agents. An example of this phenomenon is therapy response to nonHDL-C [8]. The decrease in non-HDL-C was $18.5 \%$ with FA alone, $45.8 \%$ with statin alone, and $45.3 \%$ with both therapies combined. Thus, the non-HDL C response to statin and FA therapy alone was not additive and could not predict the non-HDL-C responses to the combination of FA to statin. This was also the case for TG, and HDL-C, although not to the same degree. Thus, the addition of FA to statin is not simply the addition of the effect of these two drug therapies and in our view, these results express a unique therapy response that may be derived from in vivo interaction between the two drugs and gene variants, including the SNPs identified in this study.

At baseline, we found no association between HDL-C and ApoAI levels and the 3 SNPs (rs3741298, rs964184, and rs10750097). Importantly, there was a significant association with HDL-C and ApoA-I levels after treatment with combination therapy of FA and statins for each SNP individually as well as specific haplotypes. A different pattern was observed for TG, where the baseline significant association of these SNPs with TG disappeared after therapy.

Lai et al. showed an association between rs3135506 in the $A P O A 5$ gene with both baseline levels and change in levels following fenofibrate therapy [4]. There was no association between rs3135506 and post therapy TG and HDL-C levels in that report.

We have observed the same pattern for TG but not HDL-C and ApoA-I. A possible explanation for the difference between the two studies is the difference in populations and the presence of statin therapy in our study; their subjects had relatively normal levels of TG and HDL-C, while our subjects were required to have low HDL-C and high TG levels to qualify for the trial. The carrier frequency for rs3135506 observed by Lai et al. was lower than that observed in our study. This difference suggests that there is an enrichment of $A P O A 5$ variants in the mixed dyslipidemia population. Other studies have shown that common genetic variants in the $A P O A 5$ gene have an increased frequency in individuals with elevated TG levels [23]. In a recent sequencing study, rs964184 was shown to have a frequency of $33 \%$ in patients with severe hypertriglyceridemia, $14 \%$ in controls, and $12.1 \%$ based on HapMap information [24]. In our mixed dyslipidemia study population, rs964184 had a frequency of $19 \%$. The rs 3741298 and rs 10750097 SNPs, which both had a frequency of $26 \%$ in our study population, had frequencies of $18.6 \%$ and $25 \%$, respectively, in large databases (HapMap for rs3741298 and 1000 genomes database for rs10750097). Interestingly, these SNPs were highly associated with HDL-C response to combination therapy with FA and statins in our study. 
Although the relatively large mean increases in HDL-C observed in the current study may be related to the low HDL-C and high TG inclusion criteria for participants in the trial, we believe that enrichment of certain variants in either the $A P O A 5$ gene or other genes in individuals with mixed dyslipidemia influenced the magnitude of the HDL-C response as well. By examining SNPs in the entire APOA5-ZNF259 gene region we were able to identify specific variants that were enriched in the mixed dyslipidemia study population and had the most significant associations with combination therapy of FA and statins in that region.

It is not possible to determine whether the SNPs we identified actually have a role in the HDL-C and ApoA-I response to combination therapy with FA and statins or are markers for therapy response. Although the CGG haplotype was associated with a greater percent change in HDL-C levels with combination therapy of FA and statins, the SNPs in the haplotype are not in protein-coding regions; thus, they are not expected to affect protein structure. However, the haplotype defined by these SNPs may include regulatory elements for the $A P O A 5$ gene or adjacent genes as recently suggested by Palmen et al. and others $[25,26]$.

Following the initial discovery of the $A P O A 5$ gene, multiple studies have demonstrated its association with TG levels. Additionally, mutations in the gene have been linked to extreme TG phenotypes, and $A P O A 5$ knock-out mice were shown to be hypertriglyceridemic, while mice over-expressing the gene had decreased TG levels [27]. The Apo A-V is thought to play an important role as a co-factor for lipoprotein lipase (LPL) TG hydrolysis. Following the discovery of the APOA5 gene's importance for TG levels, a detailed characterization of its genomic region was performed which identified a number of haplotypes that were associated with TG levels [21,22]. We demonstrated that these haplotypes were associated with FA therapy response.

An association between the $A P O A 5$ gene region and CHD events was demonstrated in a large meta-analysis in which rs662799 in the $A P O A 5$ promoter was associated with both increased TG levels and CHD events [26]. Other studies have demonstrated that APOA5 variants were associated with carotid [28] and abdominal aorta atherosclerosis [29].

The ability of fenofibrate to reduce CHD has been studied in the ACCORD and FIELD randomized clinical trials which enrolled diabetic patients [2,3]. Neither trial showed cardiovascular outcome benefit for the overall study population; however, both trials showed a CHD reduction for the mixed dyslipidemia subgroups. In the ACCORD study, the primary outcome rate for the individuals with mixed dyslipidemia (TG > $204 \mathrm{mg} / \mathrm{dL}$ and HDL-C < $34 \mathrm{mg} / \mathrm{dL}$ ) subset was $12 \%$ in the fenofibrate group and $17 \%$ in the placebo group. A test for heterogeneity between individuals with mixed dyslipidemia and all other patients in that study was borderline significant $(P=0.057)$. In the FIELD study, the proportion of events was $14 \%$ in the fenofibrate group and $17.8 \%$ in the placebo group with $27 \%$ relative risk reduction for individuals with mixed dyslipidemia phenotype (TG $>2.3 \mathrm{mmol} / \mathrm{l}$ and HDL-C $<1.03 \mathrm{mmol} / \mathrm{l}$ for men and $1.29 \mathrm{mmol} / \mathrm{l}$ for women). 
In summary, genetic variants in the $A P O A 5$ gene region are associated with increased TG, increased CHD events, and greater response to combination therapy with FA and statins in patients with mixed dyslipidemia. Furthermore, genetic variants in the APOA5 region are present at an increased frequency in individuals with mixed dyslipidemia, and individuals with mixed dyslipidemia have a greater response to therapy with respect to cardiovascular risk reduction. We, therefore, postulate that genetic variants in the $A P O A 5$ region, and potentially other genetic variants, may partly explain why patients with mixed dyslipidemia have a greater benefit in therapy with fenofibrate preparations. Additional research with existing clinical trial datasets along with prospective randomized clinical trials would be required to determine whether genetic variants, might help to identify individuals with the most favorable benefit to CHD risk ratio from therapy with fenofibrate in combination with statins.

\section{Supplementary Material}

Refer to Web version on PubMed Central for supplementary material.

\section{Funding source}

DNA extraction and genotyping funded by Abbott Pharmaceuticals.

\section{References}

[1]. Ginsberg HN, Elam MB, Lovato LC, et al. Effects of combination lipid therapy in type 2 diabetes mellitus. N Engl J Med 2010;362:1563-74. [PubMed: 20228404]

[2]. Scott R, O'Brien R, Fulcher G, et al. Effects of fenofibrate treatment on cardiovascular disease risk in 9795 individuals with type 2 diabetes and various components of the metabolic syndrome: the Fenofibrate Intervention and Event Lowering in Diabetes (FIELD) study. Diabetes Care 2009;32:493-8. [PubMed: 18984774]

[3]. Keech A, Simes RJ, Barter P, et al. Effects of long-term fenofibrate therapy on cardiovascular events in 9795 people with type 2 diabetes mellitus (the FIELD study): randomised controlled trial. Lancet 2005;366:1849-61. [PubMed: 16310551]

[4]. Lai CQ, Arnett DK, Corella D, et al. Fenofibrate effect on triglyceride and post-prandial response of apolipoprotein A5 variants: the GOLDN study. Arterioscler Thromb Vasc Biol 2007;27:141725. [PubMed: 17431185]

[5]. Wojczynski MK, Gao G, Borecki I, et al. ApolipoproteinB genetic variants modify the response to fenofibrate: a GOLDN study. J Lipid Res 2010;51:3316-23. [PubMed: 20724655]

[6]. Perez-Martinez P, Corella D, Shen J, et al. Association between glucokinase regulatory protein (GCKR) and apolipoprotein A5 (APOA5) gene polymorphisms and triacylglycerol concentrations in fasting, postprandial, and fenofibrate-treated states. Am J Clin Nutr 2009;89:391-9. [PubMed: 19056598]

[7]. Jones PH, Bays HE, Davidson MH, et al. Evaluation of a new formulation of fenofibric acid, ABT-335, co-administered with statins: study design and rationale of a phase III clinical programme. Clin Drug Investig 2008;28:625-34.

[8]. Jones PH, Davidson MH, Kashyap ML, et al. Efficacy and safety of ABT-335 (fenofibric acid) in combination with rosuvastatin in patients with mixed dyslipidemia: a phase 3 study. Atherosclerosis 2009;204:208-15. [PubMed: 18996523]

[9]. van Raalte DH, Li M, Pritchard PH, Wasan KM. Peroxisome proliferator-activated receptor (PPAR)-alpha: a pharmacological target with a promising future. Pharm Res 2004;21:1531-8. [PubMed: 15497675] 
[10]. Havel RJ, Kane JP. Introduction: structure and metabolism of plasma lipoproteins In: Valle D, Beaudet AL, Vogelstein B, Kinzler KW, Antonarakis SE, Ballabio A, editors. Metabolic and molecular bases of inherited disease. 8th ed. New York: McGraw-Hill; 2001, www.ommbid.com.

[11]. Kathiresan S, Willer CJ, Peloso GM, et al. Common variants at 30 loci contribute to polygenic dyslipidemia. Nat Genet 2009;41:56-65. [PubMed: 19060906]

[12]. Willer CJ, Sanna S, Jackson AU, et al. Newly identified loci that influence lipid concentrations and risk of coronary artery disease. Nat Genet 2008;40:161-9. [PubMed: 18193043]

[13]. Spirin V, Schmidt S, Pertsemlidis A, Cooper RS, Cohen JC, Sunyaev SR. Common singlenucleotide polymorphisms act in concert to affect plasma levels of high-density lipoprotein cholesterol. Am J Hum Genet 2007;81:1298-303. [PubMed: 17952847]

[14]. Thompson A, Di Angelantonio E, Sarwar N, et al. Association of cholesteryl ester transfer protein genotypes with CETP mass and activity, lipid levels, and coronary risk. JAMA 2008;299:2777-88. [PubMed: 18560005]

[15]. Seldin MF, Shigeta R, Villoslada P, et al. European population substructure: clustering of northern and southern populations. PLoS Genet 2006;2:e143. [PubMed: 17044734]

[16]. Purcell S, Neale B, Todd-Brown K, et al. PLINK: a tool set for whole-genome association and population-based linkage analyses. Am J Hum Genet 2007;81:559-75. [PubMed: 17701901]

[17]. Koh KK, Quon MJ, Han SH, et al. Additive beneficial effects of fenofibrate combined with atorvastatin in the treatment of combined hyperlipidemia. J Am Coll Cardiol 2005;45:1649-53. [PubMed: 15893182]

[18]. Athyros VG, Papageorgiou AA, Athyrou VV, Demitriadis DS, Kontopoulos AG. Atorvastatin and micronized fenofibrate alone and in combination in type 2 diabetes with combined hyperlipidemia. Diabetes Care 2002;25: 1198-202. [PubMed: 12087019]

[19]. Grundy SM, Vega GL, Yuan Z, Battisti WP, Brady WE, Palmisano J. Effectiveness and tolerability of simvastatin plus fenofibrate for combined hyperlipidemia (the SAFARI trial). Am J Cardiol 2005;95:462-8. [PubMed: 15695129]

[20]. Vega GL, Ma PT, Cater NB, et al. Effects of adding fenofibrate (200 mg/day) to simvastatin (10 $\mathrm{mg}$ /day) in patients with combined hyperlipidemia and metabolic syndrome. Am J Cardiol 2003;91:956-60. [PubMed: 12686335]

[21]. Olivier M, Wang X, Cole R, et al. Haplotype analysis of the apolipoprotein gene cluster on human chromosome 11. Genomics 2004;83: 912-23. [PubMed: 15081120]

[22]. Pennacchio LA, Olivier M, Hubacek JA, Krauss RM, Rubin EM, Cohen JC. Two independent apolipoprotein A5 haplotypes influence human plasma triglyceride levels. Hum Mol Genet 2002;11: 3031-8. [PubMed: 12417524]

[23]. Wang J, Ban MR, Kennedy BA, et al. APOA5 genetic variants are markers for classic hyperlipoproteinemia phenotypes and hypertriglyceridemia. Nat Clin Pract Cardiovasc Med 2008;5:730-7. [PubMed: 18779834]

[24]. Johansen CT, Wang J, Lanktree MB, et al. Excess of rare variants in genes identified by genomewide association study of hypertriglyceridemia. Nat Genet 2010;42:684-7. [PubMed: 20657596]

[25]. Palmen J, Smith AJ, Dorfmeister B, Putt W, Humphries SE, Talmud PJ. The functional interaction on in vitro gene expression of APOA5 SNPs, defining haplotype APOA52, and their paradoxical association with plasma triglyceride but not plasma apoAV levels. Biochim Biophys Acta 2008;1782: 447-52. [PubMed: 18395529]

[26]. Sarwar N, Sandhu MS, Ricketts SL, et al. Triglyceride-mediated pathways and coronary disease: collaborative analysis of 101 studies. Lancet 2010;375:1634-9. [PubMed: 20452521]

[27]. Pennacchio LA, Olivier M, Hubacek JA, et al. An apolipoprotein influencing triglycerides in humans and mice revealed by comparative sequencing. Science 2001;294:169-73. [PubMed: 11588264]

[28]. Elosua R, Ordovas JM, Cupples LA, et al. Variants at the APOA5 locus, association with carotid atherosclerosis, and modification by obesity: the Framingham Study. J Lipid Res 2006;47:990-6. [PubMed: 16474174]

[29]. Laurila PP, Naukkarinen J, Kristiansson K, et al. Genetic association and interaction analysis of USF1 and APOA5 on lipid levels and atherosclerosis. Arterioscler Thromb Vasc Biol 2010;30:346-52. [PubMed: 19910639] 


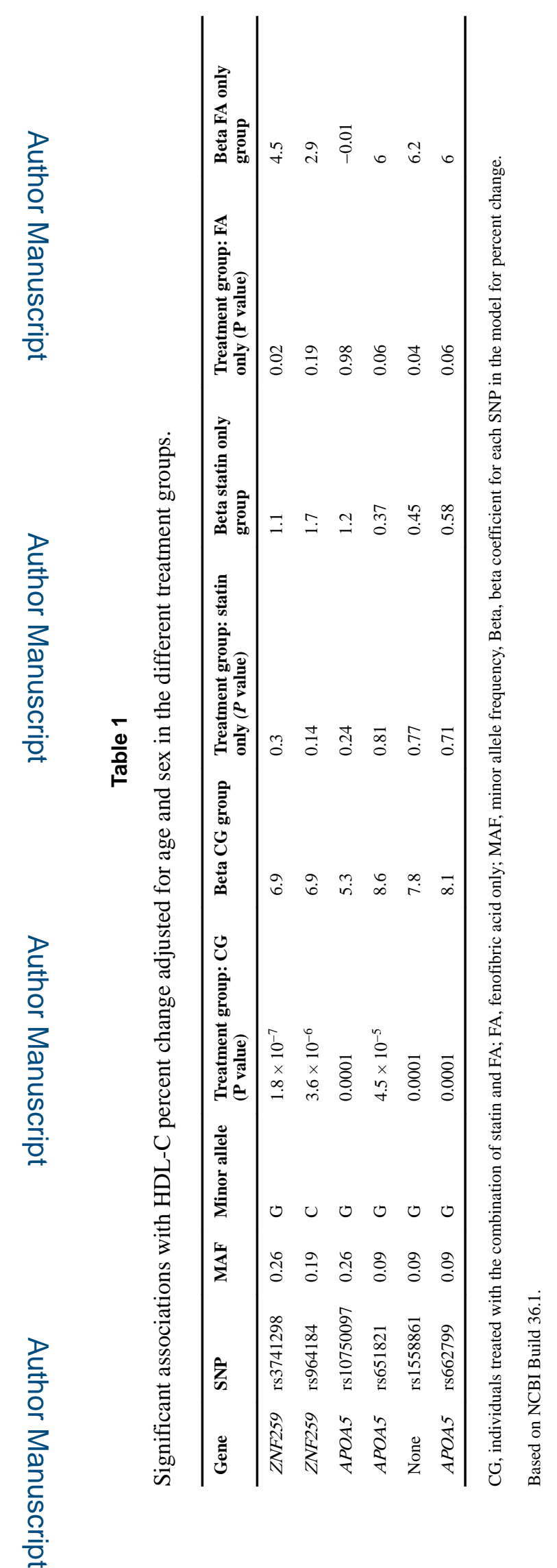

Atherosclerosis. Author manuscript; available in PMC 2018 October 08. 
Table 2

Means of percent change in HDL-C, ApoA-I, and TG after therapy for genotypes in the CG group.

\begin{tabular}{|c|c|c|c|c|c|c|}
\hline SNP & Homozygous & Heterozygous & Wild type & $P^{*}($ ANOVA) & Beta & $\mathbf{P}^{* * *}$ \\
\hline \multicolumn{7}{|l|}{ HDL-C } \\
\hline rs 964184 & $\mathrm{C} / \mathrm{C}$ & $\mathrm{C} / \mathrm{G}$ & $\mathrm{G} / \mathrm{G}$ & $1.3 \times 10^{-5}$ & 4.2 & $1.8 \times 10^{-6}$ \\
\hline Mean $(\%)$ & 29.74 & 22.87 & 15.62 & & & \\
\hline SE & 0.04 & 0.04 & 0.03 & & & \\
\hline rs3741298 & $\mathrm{G} / \mathrm{G}$ & $\mathrm{G} / \mathrm{A}$ & $\mathrm{A} / \mathrm{A}$ & $6.4 \times 10^{-7}$ & 3.6 & $1.7 \times 10^{-\varepsilon}$ \\
\hline Mean $(\%)$ & 31.7 & 20.6 & 15 & & & \\
\hline SE & 0.04 & 0.04 & 0.03 & & & \\
\hline rs 10750097 & $\mathrm{G} / \mathrm{G}$ & $\mathrm{G} / \mathrm{A}$ & $\mathrm{A} / \mathrm{A}$ & 0.0004 & 1.1 & $6.2 \times 10^{-}$ \\
\hline Mean $(\%)$ & 28.3 & 20.4 & 15.9 & & & \\
\hline SE & 0.05 & 0.03 & 0.03 & & & \\
\hline \multicolumn{7}{|l|}{ ApoA-I } \\
\hline rs964184 & $\mathrm{C} / \mathrm{C}$ & $\mathrm{C} / \mathrm{G}$ & $\mathrm{G} / \mathrm{G}$ & 0.0016 & 4.9 & $2.9 \times 10^{-}$ \\
\hline Mean $(\%)$ & 18.5 & 12.4 & 7.9 & & & \\
\hline SE & 0.04 & 0.02 & 0.01 & & & \\
\hline rs3741298 & $\mathrm{G} / \mathrm{G}$ & $\mathrm{G} / \mathrm{A}$ & $\mathrm{A} / \mathrm{A}$ & 0.0013 & 5.2 & $6.9 \times 10^{-7}$ \\
\hline Mean $(\%)$ & 15.2 & 12.1 & 7.2 & & & \\
\hline SE & 0.03 & 0.03 & 0.03 & & & \\
\hline rs 10750097 & $\mathrm{G} / \mathrm{G}$ & $\mathrm{G} / \mathrm{A}$ & $\mathrm{A} / \mathrm{A}$ & 0.039 & 3.4 & 0.001 \\
\hline Mean $(\%)$ & 15.3 & 11 & 8.2 & & & \\
\hline SE & 0.04 & 0.02 & 0.02 & & & \\
\hline \multicolumn{7}{|l|}{ TG } \\
\hline rs 964184 & $\mathrm{C} / \mathrm{C}$ & $\mathrm{C} / \mathrm{G}$ & $\mathrm{G} / \mathrm{G}$ & 0.41 & 0.5 & 0.78 \\
\hline Mean $(\%)$ & -45 & -46.7 & -43.1 & & & \\
\hline SE & 0.05 & 0.04 & 0.04 & & & \\
\hline rs3741298 & $\mathrm{G} / \mathrm{G}$ & G/A & $\mathrm{A} / \mathrm{A}$ & 0.25 & -0.8 & 0.55 \\
\hline Mean (\%) & -50.5 & -44.9 & -43 & & & \\
\hline $\mathrm{SE}$ & 0.04 & 0.04 & 0.04 & & & \\
\hline rs 10750097 & $\mathrm{G} / \mathrm{G}$ & $\mathrm{G} / \mathrm{A}$ & $\mathrm{A} / \mathrm{A}$ & 0.82 & 0.9 & 0.53 \\
\hline Mean $(\%)$ & -43.4 & -45.3 & -43.7 & & & \\
\hline SE & 0.05 & 0.04 & 0.04 & & & \\
\hline
\end{tabular}

CG, individuals receiving combination treatment with Statins and FA only; Beta, beta coefficient for each SNP in the model for percent change.

${ }^{*} P$ value for single-factor ANOVA test between the means of the three possible genotypes.

**

Multivariate regression analysis for percent change including the copy number of the minor allele, age, sex, body mass index (BMI), smoking, baseline trait level, baseline TG levels (for HDL-C only) and diabetes (adjustment for post therapy TG, or percent change in TG following therapy, did not have a major affect on the significance of the association as presented in Supplemental Table 5). 